\title{
Blood biomarkers associated with autism spectrum disorder may provide early diagnosis
}

\author{
Naila Rabbani ${ }^{1}$ and Paul J Thornalley² \\ ${ }^{1}$ Department of Basic Medical Sciences, College of Medicine, QU Health, Qatar University, PO Box 2713, Doha, Qatar \\ and 'Diabetes Research Center, Qatar Biomedical Research Institute, Hamad Bin Khalifa University, Qatar Foundation, PO Box 34110, Doha, Qatar
}

\section{BACKGROUND}

Autism Spectrum Disorders (ASD) are a collection of neuropsychiatric disorders. ASD affects 1 in 87 in Qatar and is expected to increase. The high variability and heterogeneity of the symptoms makes diagnosis of ASD difficult and uncertain, particularly at the early stages of development. If detected early, clinical support can be given to promote optimal development and well-being of children with ASD and even achieve complete remission. The current method of diagnosing ASD is by observations and interviews made by experts in child development to assess child behavior, communication and cognition: the ADOS test. There is often delay in referral for expert diagnosis; delay is typically $>18$ months in Qatar and $>4$ years in EU and USA. The diagnostic accuracy is $60-70 \%$.

Mutations in proteins transporters of amino acids have been linked to some cases of ASD. We hypothesized that levels of amino acids in blood, including amino acids damaged by modification by sugars (glycation), oxidation and nitration may provide novel biomarker for diagnosis of ASD at very young age.

\section{Problem with current diagnosis}

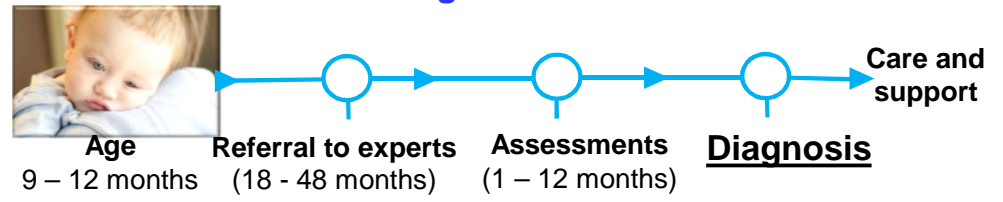

\section{Prevalence and the cost of care in Qatar}

\section{Birth: 25000 p.a. $\quad$ Care \& support} ( 1 in 87 ca. 6,600 cases) (ca. $\$ 1.6$ billion p.a. in Qatar)

Aim: To Develop a blood test for autism to provide early and swift diagnosis by measure the levels of damaged amino acids in the blood of children with ASD and children with normal development and use artificial intelligence machine learning techniques to develop diagnostic algorithms for diagnosis of ASD.

\section{METHODS}

Subject recruitment Sixty-nine children were recruited for this study: 38 had a diagnosis of ASD (29 males and 9 females) and 31 were classified as Typically Developing (TD) children (23 males and 8 females) - Figure 1. Subject age was: ASD group, 7.6 years \pm 2.0 years, range $5-12$

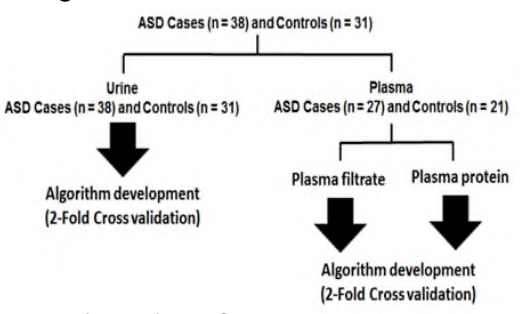

(2-Fold Crossvalidation)

years and TD group, $8.6 \pm 2.0$ years, range $5-12$ years $(P>0.05)$. All ASD subjects received a diagnosis of ASD by two child development experts at the Child Neurology and Psychiatry Unit of the Bellaria Hospital of Bologna (IRCCS Institute of Neurological Sciences), according to the Diagnostic and Statistical Manual of Mental Disorders V (DSM 5 criteria, Autism Diagnostic Observation Schedule (ADOS), Childhood Autism Rating Scale (CARS) and characteristics of onset pattern of ASD defined according to Ozonoff et al. All subjects were recruited at the Child Neurology and Psychiatry Unit of the Bellaria Hospital of Bologna, Bologna, Italy.

LC-MS/MS analysis of protein glycation and oxidation (AGEomics platform)
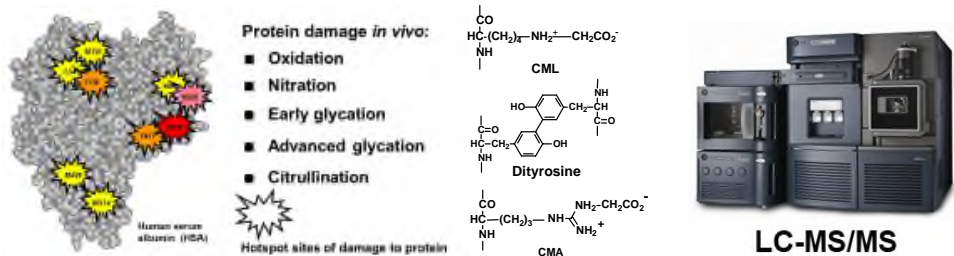

Plasma proteins undergoing continuous spontaneous modifications by sugars (glycation), oxidation and other processes. The adducts formed may be quantified in a small volume of blood by stable isotopic dilution analysis liquid chromatography-tandem mass spectrometry (LC-MS/MS). A blood plasma sample $(50 \mu \mathrm{l})$ from cases and control is processed through 4 cycles 10 -fold dilution and concentration by ultrafiltration over a $10 \mathrm{kDa}$ cut-off filter to wash the protein. The protein $1(00 \mu \mathrm{g})$ is delipidated and hydrolysed exhaustively to amino acids by successive addition of pepsin, Pronase E, Prolidase and leucine aminopeptidase over 4 days. The digest is then mixed with stable isotopic standards and analysed for ca. 20 chemically-defined protein glycation and oxidation adducts by LC-MS/MS.

Machine learning development of diagnostic algorithms: support vector machines (SVM) Use of hyperplanes and maximum margin in SVM algorithm development:

(a) Two-dimensional scatter plot for a two-classlabelled dataset of outcome, o \& + . The data can be separated by an infinite number of hyperplanes - three are shown $(\mathrm{f} 1, \mathrm{f} 2, \mathrm{f} 3)$

(b) Hyperplane maximizes the margin $(\mathrm{m})$ between the two classes of outcome, 0 and + . These are specified by the "support vectors" maximum differences from data at the margins.
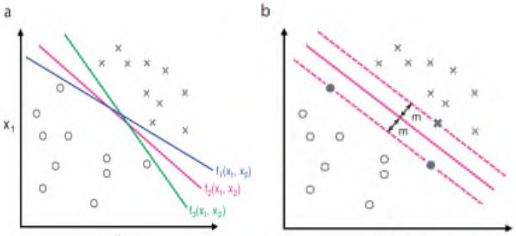

\section{RESULTS}

Changes in protein glycation and oxidation in children with autism spectrum disorder, compared to children with normal development Children with ASD had plasma protein with increased glycation adducts $-\mathrm{N}_{\varepsilon}$-carboxy methyl-lysine $(\mathrm{CML})$ and $\mathrm{N}_{\omega}$-carboxymethylarginine (CMA), and increased oxidation damage marker, dityrosine (DT), with respect to children with normal development.

A.

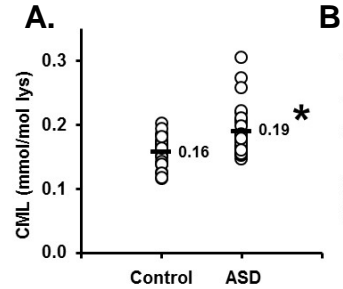

B. 0.20

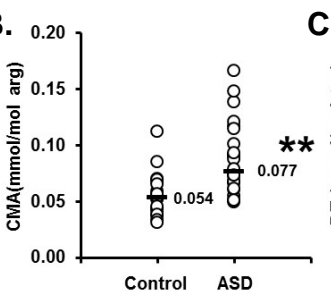

C. 0.3

$\circ$

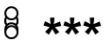
Oे

Figure 3. Increase protein glycation and oxidation adducts of plasma protein in children with ASD. A. $\mathrm{N}_{\varepsilon}$-Carboxymethyl-lysine (CML). B. $\mathrm{N}_{\omega}$-carboxy methylarginine (CMA). C. dityrosine (DT). Significance: ${ }^{*},{ }^{* *}$ and ${ }^{* * *}$, $P<0.05,, P<0.01$ and $P<0.001$, respectively; Mann-Whitney $U$ test.

Interpretation $\mathrm{CML}$ and $\mathrm{CMA}$ are both formed by protein modification by glyoxal. Increased glyoxal is typically sourced from lipid peroxidation - possibly linked to low grade inflammation. DT is mainly formed by protein modification by dual oxidase (DUOX). DUOX is increased in response to microbial challenge to gut/lung mucosal immunity. There is likely increased DUOX-mediated protein modification in plasma (and interstitial fluid) in ASD
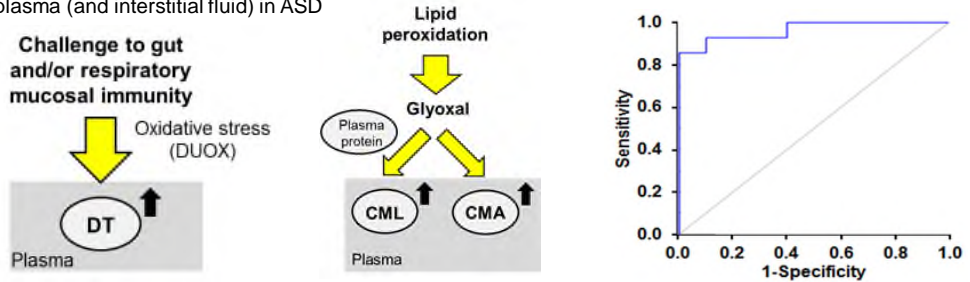

Figure 4. CML, CMA and DT association with inflammation \& immunity and Receiver operating characteristic curve for diagnostic of autism by features of plasma protein glycation and oxidation

Diagnostic algorithm development by machine learning Algorithms to discriminate between children with ASD and normal development had features: plasma protein CML, CMA, and 3deoxyglucosone-derived hydroimidazolone and DT. The sensitivity, specificity and accuracy was $92 \%, 84 \%$ and $88 \%$, respectively. The diagnostic odds ratio (DOR) was 68; cf. DOR = 7 for current ADOS test.

IP protection: Qatar University owns the world wide patent of diagnostic algorithm blood test for autism.

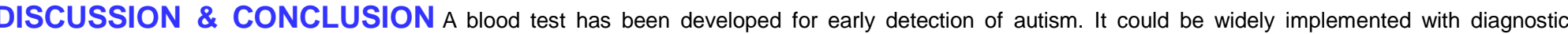

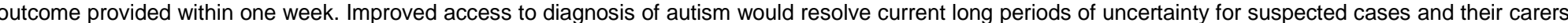

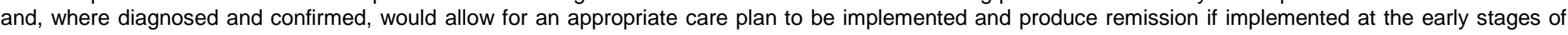

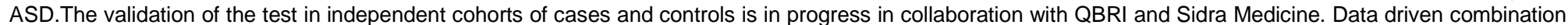

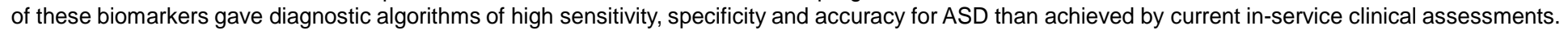

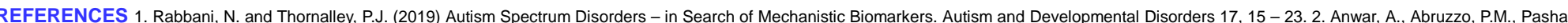

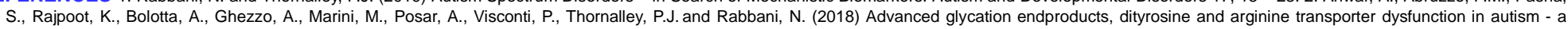
source of biomarkers for clinical diagnosis. Molecular Autism 9: 3 . 